\title{
EFICÁCIA DE MISTURAS DE HERBICIDAS NO CONTROLE DE UM BIÓTIPO DE Euphorbia heterophylla TOLERANTE AOS HERBICIDAS INIBIDORES DA ACETOLACTATO SINTASE, NA CULTURA DA SOJA
}

\author{
Erivelton S. Roman ${ }^{1}$ \\ ${ }^{1}$ Eng $^{\circ}$. Agr ${ }^{\circ}$., Ph. D., Pesquisador. Embrapa Trigo. Caixa Postal 451. Passo Fundo, RS 99001-970 eroman@enpt.embrapa.br
}

\begin{abstract}
RESUMO
Um experimento foi conduzido em condições de campo, cujo objetivo foi verificar a eficácia dos herbicidas isolados lactofen, imazethapyr, chlorimuron-ethyl, cloransulam-metil, oxasulfuron, flumiclorac-pentil aplicados isoladamente e em mistura com lactofen, com pós-emergência, no controle de Euphorbia heterophylla tolerante aos inibidores da ALS, na cultura de soja. O biótipo de E. heterophylla não foi controlado por imazethapyr aplicado isoladamente e não apresentou nenhum sintoma fitotóxico, mesmo na dose de $1,5 \mathrm{vez}$ a dose recomendada. Da mesma forma, não foram observados sintomas de danos em plantas desse biótipo quando chlorimuron-ethyl foi aplicado na dose de $120 \mathrm{~g} / \mathrm{ha}$, no teste realizado em condições controladas, o que sugere ser provável a existência de tolerância também a este composto com semelhante mecanismo de ação. O tratamento com melhor controle final, em termos absolutos, foi alcançado com lactofen $(120 \mathrm{~g} / \mathrm{ha})+$ Attach $(0,2 \%)$ (em aplicação sequencial). Entretanto, os tratamentos lactofen+imazethapyr $(120+50 \mathrm{~g} / \mathrm{ha})$, lactofen+oxasulfuron $(120+45 \mathrm{~g} / \mathrm{ha})$, lactofen+flumiclorac-pentil $(120+40 \mathrm{~g} / \mathrm{ha})$, lactofen $(120 \mathrm{~g} / \mathrm{ha})+$ Attach $(0,2 \%)$, lactofen $(120 \mathrm{~g} / \mathrm{ha})$, lactofen+cloransulam-metil $(120+30 \mathrm{~g} / \mathrm{ha})$ e lactofen+chlorimuron-ethyl $(120+10 \mathrm{~g} / \mathrm{ha})$ não apresentaram diferenças significativas entre si ao nível de $5 \%$ de probabilidade. Os controles finais (testemunhas) foram apenas regulares, indicando a necessidade de estudos adicionais para verificar em que condições as misturas desses herbicidas podem ser recomendadas, haja vista a possibilidade de resistência ao imazethapyr e ao chlorimuron-ethyl, componentes de algumas misturas.
\end{abstract}

Palavras-chaves: tolerância, imidazolinonas, sulfonilureias.

\section{ABSTRACT \\ Efficacy of herbicide mixtures in the control of an Euphorbia heteropylla biotype tolerant to acetolactate synthase in soybean}

An experiment was conducted under field conditions to study the efficacy of the herbicides lactofen, imazethapyr, chlorimuron-ethyl, cloransulam-metil, oxasulfuron, and flumiclorac-pentil, alone and in combination with lactofen, for $E$. heterophylla control in soybeans. The E. heterophylla biotype was supposed to be tolerant to herbicides whose mechanism of action is on ALS enzyme inhibition. The best treatment in absolute terms was lactofen at $120 \mathrm{~g}$ /ha plus Attach $0.2 \%$ as sequential applications. However, lactofen + imazethapyr $(120+50 \mathrm{~g} / \mathrm{ha})$, lactofen + oxasulfuron $(120+45 \mathrm{~g} / \mathrm{ha})$, lactofen + flumiclorac-pentil $(120+40 \mathrm{~g} / \mathrm{ha})$, lactofen $(120 \mathrm{~g} / \mathrm{ha})+$ Attach $0.2 \%$, lactofen $(120 \mathrm{~g} / \mathrm{ha})$, lactofen + cloransulam-methyl $(120+30 \mathrm{~g} / \mathrm{ha})$ and lactofen + chlorimuron-ethyl $(120+10 \mathrm{~g} / \mathrm{ha})$ were not statiscally different $(5 \%$ probability $)$. It should be pointed out that the control of the biotype was considered medium, therefore indicating that additional studies should be 
carried out to verify in which conditions mixtures of protox inhibitors with ALS inhibiting herbicides should be recommended considering the tolerance of the biotype to imazethapyr and chorimuron-ethyl that were components of some herbicide mixtures.

Key words: tolerance, imidazolinones, sulfonilureas.

\section{INTRODUÇÃO}

Até recentemente, as preocupações com o desenvolvimento de resistência a defensivos agrícolas estavam mais relacionadas com o uso de fungicidas e de inseticidas. Desde a resistência de Senecio vulgaris aos herbicidas da classe das triazinas, relatada por Ryan (1970), o aumento no número de biótipos resistentes a uma ou mais classes de herbicidas vem crescendo, sendo o fenômeno relatado em vários locais do mundo (Gressel \& Segel, 1990; Powles \& Howat, 1990; Heap, 1997).

Algumas classes de herbicidas, embora estruturalmente diferentes, como os pertencentes ao grupo da imidazolinonas e sulfoniluréias, têm o mesmo mecanismo de ação, inibindo a enzima ALS (acetolactato sintase) (Shaner, 1991) e, em conseqüência, a síntese da valina, leucina e isoleucina (Holt et al., 1993), aminoácidos essenciais ao crescimento e ao desenvolvimento de plantas. A falta de um deles pode ser letal para muitas espécies (Holt et al., 1993).

Os herbicidas inibidores da ALS são usados, no Brasil, principalmente para o controle de plantas daninhas dicotiledôneas em soja. A sua seletividade é baseada, principalmente, na capacidade da planta em metabolizar rapidamente o herbicida, formando compostos não fitotóxicos. Devido à sua aplicação repetida, muitas espécies de plantas daninhas que eram suscetíveis a esses produtos desenvolveram biótipos resistentes, através de mutação e seleção natural (Saari et al., 1992). No Brasil já foi relatada a ocorrência de biótipos de Euphorbia heterophylla e de Bidens sp. resistentes (Christoffoleti et al., 1997). Gazziero et al. (1998) também confirmaram a ocorrência de resistência cruzada aos inibidores de ALS em populações de Euphorbia heterophylla, infestantes em lavouras de soja do Paraná.

As misturas e o uso alternado de ingredientes ativos com diferentes mecanismos de ação estão entre as alternativas para manejo da resistência de plantas daninhas a herbicidas (Christoffoleti et al., 1994).

O uso de herbicidas cujo mecanismo de ação é a inibição da enzima protox, uma das enzimas que atuam na síntese de clorofila, é uma alternativa para o manejo da resistência aos inibidores da ALS. Esses herbicidas causam acumulação de protoporfirina IX (protox), que, em presença de luz e de oxigênio molecular, gera oxigênio elementar, o qual causa destruição da membrana celular, resultando em rápida dessecação e necrose de tecidos. Possuem modo de ação essencialmente de contato (Lydon \& Duke, 1988). Não são conhecidas espécies resistentes aos herbicidas inibidores da protox (Duke et al., 1996), de forma que esses herbicidas podem ser importantes em programas de manejo de plantas daninhas. Por apresentarem modos e mecanismos de ação diferentes, o emprego de misturas em tanque de herbicidas do grupo das imidazolinonas e dos difenil-éteres apresentase como possível estratégia no manejo da resistência de plantas daninhas a herbicidas, na cultura de soja.

O objetivo deste trabalho foi verificar a eficácia dos produtos isolados e de suas misturas, aplicados em pós-emergência, no controle de E. heterophylla tolerante aos inibidores da ALS, na cultura de soja.

\section{MATERIAL E MÉTODOS}

O experimento foi conduzido na propriedade do sr. Augusto Trein, localizada no município de Passo Fundo, RS, no ano agrícola 1999/2000, em Latossolo Roxo distrófico. A cultivar de soja reagente foi a RS-10, com 40 plantas $/ \mathrm{m}^{2}$, semeada no espaçamento de $0,5 \mathrm{~m}$ entre linhas. A semeadura foi realizada em sistema de plantio direto, no dia 22 de dezembro de 1999.

Utilizou-se um biótipo de E. heterophylla que apresentou falhas de controle em campo, com a aplicação de herbicidas inibidores de ALS. Estes herbicidas foram usados por mais de 10 anos na referida propriedade rural. Devido a redução da eficiência de controle, suspeita-se de resistência deste biótipo aos herbicidas com tal mecanismo de ação.

Os tratamentos (Tabela 1) foram aplicados no dia 19 de janeiro de 2000, com início da pulverização às 16:00 h e término às 17:45 h. Para verificar a possibilidade de resistência cruzada do biótipo a outro herbicida, realizou-se um teste em condições controladas com chlorimuron-ethyl, na dose de $120 \mathrm{~g} / \mathrm{ha}$. Nesse momento, a temperatura do ar era de $22{ }^{\circ} \mathrm{C}$ e a umidade relativa de $66 \%$. A aplicação foi realizada usando-se um pulverizador costal de precisão, com pressão de trabalho de $1,1 \mathrm{~kg} / \mathrm{cm}^{2}$ proporcionada por gás carbônico, munido de bicos de jato em forma de leque, tipo 110.015, espaçados $0,5 \mathrm{~m}$ entre si e posicionados à altura de $50 \mathrm{~cm}$ acima das plantas. O volume de calda usado foi de 100 1/ha. O delineamento experimental foi de blocos ao acaso, com 4 repetições. As parcelas mediam $3 \mathrm{~m} \times 7 \mathrm{~m}$.

No momento da aplicação dos tratamentos o estádio de desenvolvimento da soja era de 2 a 3 trifólios. A planta daninha (Euphorbia heterophylla) estava no estádio de crescimento de 2 a 5 folhas, com densidade populacional de cerca de 500 plantas $/ \mathrm{m}^{2}$. 
Eficácia de misturas de herbicidas...

Tabela 1. Tratamentos aplicados em pós-emergência no controle de Euphorbia heterophylla. Passo Fundo, RS. $1999 / 2000$.

\begin{tabular}{|c|c|c|c|}
\hline \multicolumn{2}{|c|}{ Tratamento } & \multicolumn{2}{|c|}{ Dose } \\
\hline Nome Técnico & Nome Comercial & $\begin{array}{c}\text { i.a. } \\
\text { (g/ha) }\end{array}$ & $\begin{array}{l}\text { Produto Comercial } \\
\text { (por hectare) }\end{array}$ \\
\hline Testemunha & - & - & - \\
\hline Imazethapyr & Pivot & 50 & 0,51 \\
\hline Im azethapyr & Pivot & 150 & 1,51 \\
\hline Chlorimuron-ethyl & Classic & 10 & $40,0 \mathrm{~g}$ \\
\hline Cloransulam-metil & Pacto & 30 & $35,7 \mathrm{~g}$ \\
\hline Oxasulfuron & Chart & 45 & $60,0 \mathrm{~g}$ \\
\hline Lactofen & Cobra & 120 & 0,51 \\
\hline Flumiclorac-pentil & Radiant & 40 & 0,41 \\
\hline Lactofen + chlorim uron-ethyl & Cobra+Classic & $120+10$ & $0,5 \mathrm{l}+40,0 \mathrm{~g}$ \\
\hline Lactofen + im azethapyr & Cobra+Pivot & $120+50$ & $0,51+0,51$ \\
\hline Lactofen +cloransulam-metil & Cobra+Pacto & $120+30$ & $0,5 \mathrm{l}+35,7 \mathrm{~g}$ \\
\hline Lactofen + oxasulfuron & Cobra + Chart & $120+45$ & $0,51+60,0 \mathrm{~g}$ \\
\hline Lactofen +flum iclorac-pentil & Cobra+Radiant & $120+40$ & $0,51+0,41$ \\
\hline Lactofen ${ }^{1}$ & Cobra & 120 & 0,501 \\
\hline Lactofen ${ }^{1}$ & Cobra & 60 & 0,251 \\
\hline
\end{tabular}

${ }^{1}$ Adicionado óleo mineral Attach a $0,2 \%$.

A eficácia dos tratamentos no controle de $E$. heterophylla foi determinada aos 7,40 e 70 dias após o tratamento (DAT) pelo método de avaliação visual, atribuindo-se notas em percentagem de controle em relação à testemunha. A escala empregada variou entre 0 (sem danos visíveis) e $100 \%$ (morte total de plantas). A avaliação da toxicidade às plantas de soja foi realizada visualmente aos 10 e aos 20 DAT, e os resultados expressos em percentagem de danos, tomando como base a redução do crescimento, a clorose e a queima da parte aérea foliar (Lich et al., 1997).

Os dados de eficácia dos tratamentos, depois de submetidos a testes de homogeneidade da variância e da distribuição do erro experimental (normalidade), foram transformados por meio de de arco seno $[(\sqrt{\mathrm{x}+1}) / 100]$, para análise de variância e para comparação das médias dos tratamentos pelo teste de Duncan, a $5 \%$ de probabilidade de erro.

Os dados de rendimento de grãos de soja, obtidos na área útil de $10 \mathrm{~m}^{2}$ em cada unidade experimental, foram submetidos à análise de variância e as médias comparadas pelo teste de Duncan, ao nível de $5 \%$ de probabilidade de erro.

\section{RESULTADOS E DISCUSSÃO}

\section{Fitotoxicidade}

Com $33,7 \%$ e $35 \%$ de dano foliar nas plantas de soja, os tratamentos de lactofen com adição de óleo mineral, pro- porcionaram a maior fitotoxicidade em termos absolutos. Lactofen, em suas misturas com os demais ingredientes ativos testados, causaram fitotoxicidade em níveis que variaram de 27,5 a 30,0\% (Tabela 2).

Os sintomas de toxicidade as plantas de soja causadas pelas aplicações dos produtos foram identificadas pelo encrespamento/enrolamento e queima da área foliar, principalmente em folhas jovens, como também relatado por Ashton \& Crafts (1981). Aos 20 DAT, as plantas de soja, na maioria dos tratamentos com misturas de herbicidas, estavam se recuperando dos sintomas presentes na avaliação anterior, estando estes evidenciados tão somente nas folhas mais velhas.

\section{Controle}

O biótipo de E. heterophylla não foi controlado por imazethapyr aplicado isoladamente e não apresentou nenhum sintoma fitotóxico, mesmo na dose de 1,5 vez a dose recomendada (Tabela 3). Da mesma forma, não foram observados sintomas de danos em plantas desse biótipo quando chlorimuron-ethyl foi aplicado na dose de $120 \mathrm{~g} / \mathrm{ha}$, no teste realizado em condições controladas. Possivelmente existe uma tolerância a este composto também com semelhante mecanismo de ação. Isto sugere que o aumento da dose desse produto não melhora o controle da espécie (Hinz \& Owen, 1997). Os herbicidas chlorimuron-ethyl (10 g/ha), cloransulammethyl (30 g/ha) e oxasulfuron (45 g/ha), quando aplicados isoladamente não proporcionaram controle de Euphorbia heterophylla nas avaliações realizadas aos 7, 40 e 70 DAT 
Erivelton S. Roman

Tabela 2. Toxicidade causada à soja aos 10 e 20 dias após os tratamentos (DAT). Passo Fundo, RS. 1999/2000.

\begin{tabular}{|c|c|c|c|}
\hline \multirow{2}{*}{ Tratamento } & \multirow{2}{*}{$\begin{array}{c}\text { Dose } \\
(\mathrm{g} / \mathrm{ha})\end{array}$} & \multicolumn{2}{|c|}{ Fitotoxicidade (\%) } \\
\hline & & $10 \mathrm{DAT}$ & $20 \mathrm{DAT}$ \\
\hline Testemunha & & 0,0 & 0,0 \\
\hline Im azethapyr & 50 & 0,0 & 0,0 \\
\hline Imazethapyr & 150 & 12,5 & 0,0 \\
\hline Chlorimuron-ethyl & 10 & 10,0 & 0,0 \\
\hline Cloransulam-metil & 30 & 16,2 & 0,0 \\
\hline Oxasulfuron & 45 & 12,5 & 3,7 \\
\hline Lactofen & 120 & 22,5 & 5,0 \\
\hline Flum iclorac-pentil & 40 & 23,7 & 3,7 \\
\hline Lactofen + ch lorim uron-ethyl & $120+10$ & 27,5 & 3,7 \\
\hline Lactofen + im azethapyr & $120+50$ & 27,5 & 3,7 \\
\hline Lactofen + cloransulam -m etil & $120+30$ & 26,2 & 5,0 \\
\hline Lactofen + oxasulfuron & $120+45$ & 27,5 & 3,7 \\
\hline Lactofen + flum iclorac-pentil & $120+40$ & 30,0 & 3,7 \\
\hline Lactofen + Attach $0,2 \%$ & 120 & 35,0 & 3,7 \\
\hline Lactofen+Attach $0,2 \%$ (sequencial) & 60 & 33,7 & 5,0 \\
\hline
\end{tabular}

Tabela 3. Controle de Euphorbia heterophylla aos 7, 40 e 70 dias após os tratamentos (DAT). Embrapa Trigo, Passo Fundo, RS. 1999-2000.

\begin{tabular}{|c|c|c|c|c|}
\hline \multirow{2}{*}{ Tratamento } & \multirow{2}{*}{$\begin{array}{c}\text { Dose } \\
\text { (g/ha) }\end{array}$} & \multicolumn{3}{|c|}{ Controle $(\%)$} \\
\hline & & 7 DAT & $40 \mathrm{DAT}$ & $70 \mathrm{DAT}$ \\
\hline Testemunha & & $0 \mathrm{e}$ & $0 \mathrm{f}$ & $0 \mathrm{c}$ \\
\hline Im azethapyr & 50 & $0 \mathrm{e}$ & $0 \mathrm{f}$ & $0 \mathrm{c}$ \\
\hline Im azethapyr & 150 & $0 \mathrm{e}$ & $0 \mathrm{f}$ & $0 \mathrm{c}$ \\
\hline Chlorim uron-ethyl & 10 & $0 \mathrm{e}$ & $0 \mathrm{f}$ & $0 \mathrm{c}$ \\
\hline Cloransulam-metil & 30 & $0 \mathrm{e}$ & $0 \mathrm{f}$ & $0 \mathrm{c}$ \\
\hline Oxasulfuron & 45 & $0 \mathrm{e}$ & $0 \mathrm{f}$ & $0 \mathrm{c}$ \\
\hline Lactofen & 120 & $84 \mathrm{abc}$ & $65 \mathrm{bc}$ & $56 \mathrm{ab}$ \\
\hline Flumiclorac-pentil & 40 & $70 \mathrm{~cd}$ & 42 ed & $42 \mathrm{~b}$ \\
\hline Lactofen + chlorimuron-ethyl & $120+10$ & $68 \mathrm{~d}$ & $38 \mathrm{e}$ & $50 \mathrm{ab}$ \\
\hline Lactofen + imazethapyr & $120+50$ & $84 \mathrm{abc}$ & $52 \mathrm{cde}$ & $64 \mathrm{ab}$ \\
\hline Lactofen+cloransulam-m etil & $120+30$ & $82 \mathrm{abc}$ & $58 \mathrm{bcd}$ & $55 \mathrm{ab}$ \\
\hline Lactofen+oxasulfuron & $120+45$ & 72 bcd & $62 \mathrm{bc}$ & $59 \mathrm{ab}$ \\
\hline Lactofen+flum iclorac-pentil & $120+40$ & $84 \mathrm{abc}$ & $64 \mathrm{bc}$ & $64 \mathrm{ab}$ \\
\hline Lactofen + Attach $0,2 \%$ & 120 & $86 \mathrm{ab}$ & $70 \mathrm{ab}$ & $58 \mathrm{ab}$ \\
\hline Lactofen + Attach $0,2 \%$ (seq.) & 60 & $91 \mathrm{a}$ & 82 a & $67 \mathrm{a}$ \\
\hline C.V. $(\%)$ & & 9,7 & 16,1 & 18,4 \\
\hline
\end{tabular}

(dias após aplicação dos tratamentos).

Os melhores tratamentos, na avaliação visual realizada aos 7 DAT, foram lactofen $(60 \mathrm{~g} / \mathrm{ha})+$ Attach a $0,2 \% \mathrm{v} . /$ v.(sequencial), lactofen (120 g/ha)+Attach a $0,2 \% \mathrm{v} . / \mathrm{v}$., lactofen + imazethapyr $(120+50 \mathrm{~g} / \mathrm{ha})$, lactofen+flumicloracpentil $(120+40 \mathrm{~g} / \mathrm{ha})$, lactofen $(120 \mathrm{~g} / \mathrm{ha}) \mathrm{e}$ lactofen+cloransulam-metil $(120+30 \mathrm{~g} / \mathrm{ha})$. Esses tratamentos apresentaram níveis de controle acima de $80 \%$ e não apresentaram diferenças significativas entre eles. Os demais tratamentos apresentaram níveis de controle inferiores a $80 \%$ (Tabela 3).
Na avaliação visual realizada aos 40 DAT, os melhores tratamentos foram lactofen $(120 \mathrm{~g} / \mathrm{ha})+$ Attach a $0,2 \%$ v./v. (sequencial), com controle de $82 \%$ e lactofen $(120 \mathrm{~g} / \mathrm{ha})$ + Attach a $0,2 \%$ v./v., com controle de $70 \%$, os quais não apresentaram diferença significativa entre si. Os demais tratamentos apresentaram controle inferior a 70\% (Tabela 3).

Na avaliação visual realizada aos 70 DAT, o tratamento com melhor controle, em termos absolutos, foi proporcionado por lactofen $(120 \mathrm{~g} / \mathrm{ha})+$ Attach a $0,2 \%$ (em aplicação sequencial) (Tabela 3). Entretanto, os tratamentos lactofen + imazethapyr $(120+50 \mathrm{~g} / \mathrm{ha})$, lactofen + oxasulfuron $(120+45$ 
$\mathrm{g} / \mathrm{ha})$, lactofen + flumiclorac-pentil $(120+40 \mathrm{~g} / \mathrm{ha})$, lactofen $(120 \mathrm{~g} / \mathrm{ha})+$ Attach a $0,2 \%$, lactofen $(120 \mathrm{~g} / \mathrm{ha})$, lactofen + cloransulam-metil $(120+30 \mathrm{~g} / \mathrm{ha})$ e lactofen + chlorimuronethyl $(120+10 \mathrm{~g} / \mathrm{ha})$ não apresentaram diferenças significativas entre si.

\section{Rendimento de grãos}

Os rendimentos de grãos variaram de $1030 \mathrm{~kg} / \mathrm{ha}$, na testemunha não capinada, a $2009 \mathrm{~kg} / \mathrm{ha}$ no tratamento com lactofen $(60 \mathrm{~g} / \mathrm{ha})+$ Attach $0,2 \%$ v./v., aplicação sequencial (Tabela 4). Os tratamentos com imazethapyr, nas doses de 50 e $150 \mathrm{~g} / \mathrm{ha}$, chlorimuron-ethyl (10 g/ha), cloransulam-metil $(30 \mathrm{~g} / \mathrm{ha})$ e oxasulfuron $(45 \mathrm{~g} / \mathrm{ha})$ apresentaram os menores rendimentos de grãos e igualaram-se estatisticamente à testemunha não capinada.

Os melhores rendimentos de grãos foram obtidos nos tratamentos com lactofen na dose de $60 \mathrm{~g} / \mathrm{ha}$ (com óleo mineral), este em aplicação seqüencial, lactofen + flumicloracpentil $(120+40 \mathrm{~g} / \mathrm{ha})$, lactofen $(120 \mathrm{~g} / \mathrm{ha})$, lactofen $(120 \mathrm{~g} /$ ha) + Attach a $0,2 \% \mathrm{v} . / \mathrm{v}$. e lactofen $(120 \mathrm{~g} / \mathrm{ha})+$ cloransulammetil (120+30 g/ha), que não apresentaram diferenças significativas entre si. Portanto, houve relação direta entre o controle da espécie e o rendimento de grãos de soja.

\section{CONCLUSÕES}

Os melhores controles de E. heterophylla foram obtidos por lactofen $(120 \mathrm{~g} / \mathrm{ha})+$ Attach $0,2 \%$ (em aplicação sequencial) e pelas misturas de lactofen + imazethapyr $(120+50 \mathrm{~g} / \mathrm{ha})$, lactofen + oxasulfuron $(120+45 \mathrm{~g} / \mathrm{ha})$, lactofen + flumiclorac-pentil $(120+40 \mathrm{~g} / \mathrm{ha})$, lactofen $(120 \mathrm{~g} / \mathrm{ha})+$ Attach $0,2 \%$, lactofen (120 g/ha), lactofen + cloransulammetil $(120+30 \mathrm{~g} / \mathrm{ha})$ e lactofen + chlorimuron-ethyl $(120+10$ $\mathrm{g} / \mathrm{ha}$ ), que não apresentaram diferença significativa entre si. No entanto, deve ser salientado que os controles finais (testemunhas) foram apenas regulares, indicando a necessidade de estudos adicionais para se verificar em que condições as misturas desses herbicidas podem ser recomendadas, tendo em vista a possibilidade de resistência ao imazethapyr e ao chlorimuron-ethyl, herbicidas componentes de alguma misturas.

\section{LITERATURA CITADA}

ASHTON, F. M.; CRAFTS, A.S. Mode of action of herbicides. New York: Wiley-Interscience, 1991. 525 p.

CHRISTOFFOLETI, P. J.; VICTORIA FILHO, R.; SILVA, C. B. Resistência de plantas daninhas aos herbicidas. Planta Daninha, v. 12, n. 1, p. 13-20, 1994.

CHRISTOFFOLETI, P. J.; VICTORIA FILHO, R.; MONQUEIRO, P. A. Resistência cruzada a herbicidas alternativos de controle de biótipos de Bidens pilosa resistentes a herbicidas inibidores de ALS. In: CONGRESSO BRASILEIRO DE CIÊNCIA DAS PLANTAS DANINHAS, 20. Caxambú, p. 64, 1997.

Tabela 4. Rendimento de grãos de soja em resposta à aplicação de herbicidas em pós-emergência, em Euphorbia heterophylla resistente.Embrapa Trigo, Passo Fundo, RS. 1999-2000.

\begin{tabular}{lcc}
\hline \multicolumn{1}{c}{ Tratamento } & $\begin{array}{c}\text { Dose } \\
(\mathbf{g} / \mathbf{h a})\end{array}$ & $\begin{array}{c}\text { Rendimento de Grãos } \\
\text { (kg/ha) }\end{array}$ \\
\hline Testemunha & - & $1170 \mathrm{~d}$ \\
Imazethapyr & 50 & $1111 \mathrm{~d}$ \\
Imazethapyr & 150 & $1030 \mathrm{~d}$ \\
Chlorimuron-ethyl & 10 & $1095 \mathrm{~d}$ \\
Cloransulam-metil & 30 & $1208 \mathrm{~d}$ \\
Oxasulfuron & 45 & $1227 \mathrm{~d}$ \\
Lactofen & 120 & $1887 \mathrm{abc}$ \\
Flumiclorac-pentil & 40 & $1604 \mathrm{bc}$ \\
Lactofen+chlorimuron-ethyl & $120+10$ & $1594 \mathrm{c}$ \\
Lactofen+imazethapyr & $120+50$ & $1680 \mathrm{bc}$ \\
Lactofen+cloransulam-metil & $120+30$ & $1789 \mathrm{abc}$ \\
Lactofen+oxasulfuron & $120+45$ & $1641 \mathrm{bc}$ \\
Lactofen+flumiclorac-pentil & $120+40$ & $1913 \mathrm{ab}$ \\
Lactofen+Attach 0,2\% & 120 & $1864 \mathrm{abc}$ \\
Lactofen+Attach 0,2\% (sequencial) & 60 & $2009 \mathrm{a}$ \\
\hline C. V. (\%) & & 6,2 \\
\hline
\end{tabular}


DUKE, S. O., DAYAN, F. E.; YAMAMOTO, M.; DUKE, M. V.; REDDY, K. N. Protoporphyrinogen oxidase inhibitors - their current and future role. INTERNATIONAL WEED CONTROL CONGRESS, Proceedings... 3: 775-780, 1996.

GAZZIERO, D. L. P.; BRIGHENTI, A. M.; MACIEL, C. G.; CHRISTOFFOLETI, P. J.; ADEGAS, F. S.; VOLL, E. Resistência de amendoim-bravo aos herbicidas da enzima ALS. Planta Daninha, v. 16, n. 2, p. 117-125, 1998.

GRESSEL, J.; SEGEL, L. A. Modeling the effectiveness of herbicide rotations and mixtures as strategies to delay or preclude resistance. Weed Technology, v. 4, p. 186-198, 1990.

HEAP, I. M. The occurrence of herbicide resistant weeds worldwide. Pesticide Science, v. 51, p. 235-243. 1997.

HINZ, J. R. R.; OWEN, M. D. K. Acetolactate synthase resistance in a common waterhemp (Amaranthus rudis) population. Weed Technology, v.11, p. 13-18. 1997.

HOLT, J. S.; POWLES, S. B.; HOLTUM, J. A. M. 1993. Mechanisms and agronomic aspects of herbicide resistance. Ann. Rev. Plant Physiol. Plant Mol. Biol., 44:
203-229. 1993.

LICH, J. M.; RENNER, K. A.; PENNER, D. 1997. Interaction of glyphosate with postemergence soybean (Glyphosate $\max$ ) herbicides. Weed Science, v. 45, p. 12-21.

LYDON, J.; DUKE, O.LS. Porphyrin synthesis is required for photobleaching activity of the $p$-nitro substituted diphenyl ether herbicides. Pestic. Biochem. Physiol, v. 36, p. 300-307. 1988.

POWLES, S. B.; HOWAT, P. D. Herbicide resistant weeds in Australia. Weed Technology, v. 4, p. 178-185. 1990.

RYAN, G. F. Resistance of common groundsel to simazine and atrazine. Weed Science, v. 18, p. 614-616. 1970.

SAARI, L. L.; COTTERMAN, J. C.; SMITH, W. S.; PRIMIANI, M. M. Sulfonylurea herbicide resistance in common chickweed, perennial ryegrass, and Russian thistle. Pestic. Biochem. Physiol, v. 42, p. 110-118. 1992.

SHANER, D. L. Mechanisms of resistance to acetolactate synthase/acetohydroxiacid synthase inhibitors. In: CASELEY, G.W.; ATKIN, R.K. (ed.). Herbicide resistance in weeds and crops. Oxford, England: Butterworth-Heinemann, p. 187-198. 1991. 